\title{
Prognostic Factors of Functional Outcome Assessed by Using the Modified Rankin Scale in Subacute Ischemic Stroke
}

\author{
Siriphan Kongsawasdi ${ }^{\mathrm{a}}$, Jakkrit Klaphajone ${ }^{\mathrm{b}, \mathrm{d}}$, Pakorn Wivatvongvana ${ }^{\mathrm{b}}$, \\ Kanokwan Watcharasaksilp ${ }^{c}$
}

\begin{abstract}
Background: Most stroke survivors spent their lifetime with disability which not only affects the clients themselves and the family but also brings economic cost to the country. Therefore, this retrospective cohort study aimed to identify independent prognostic determinants associated with functional recovery in ischemic stroke within 6 months after onset.
\end{abstract}

Methods: Data from all first-onset ischemic stroke patients admitted to the acute stroke unit of the tertiary, university hospital were reviewed for 5 years consecutively. The functional outcome of the patients was recorded during 6-month follow-up by using the modified Rankin Scale (mRS). Baseline characteristics, motor assessment and all stroke-related variables were assessed during first week after stroke and 6-month follow-up. In order to derive clinical predictors, the backward stepwise multivariable risk regression analyses were used with the generalized linear model.

Results: The result revealed that in the 358 patients recruited into this study, $255(71.2 \%)$ were in the functional recovery group (mRS score of 1 - 3) within 6 months after onset. The final model of multivariable risk regression analysis, with generalized linear model, demonstrated that the independent variables of functional recovery were leg score with a risk ratio $(\mathrm{RR}=1.92,95 \%$ confidence interval $(\mathrm{CI}): 1.14-3.21$, $\mathrm{P}=0.013)$, arm score $(\mathrm{RR}=1.75,95 \% \mathrm{CI}: 1.02-3.01, \mathrm{P}=0.042)$ and age older than 75 years $(\mathrm{RR}=1.36,95 \% \mathrm{CI}: 1.04-1.77, \mathrm{P}=0.025)$.

Conclusions: Achieving functional recovery during 6 months post stroke was related to age and motor improvement. With limited re-

Manuscript submitted February 26, 2019, accepted March 16, 2019

${ }^{a}$ Clinical Epidemiology Program, Faculty of Medicine, Chiang Mai University, Chiang Mai, Thailand

bepartment of Rehabilitation Medicine, Faculty of Medicine, Chiang Mai University, Chiang Mai, Thailand

'Department of Internal Medicine, Faculty of Medicine, Chiang Mai University, Chiang Mai, Thailand

${ }^{\mathrm{d} C}$ Corresponding Author: Jakkrit Klaphajone, Department of Rehabilitation Medicine, Faculty of Medicine, Chiang Mai University, 110 Intawaroros Road, Muang District, Chiang Mai 50200, Thailand.

Email: makodica@yahoo.com

doi: https://doi.org/10.14740/jocmr3799 sources, continuity of rehabilitation training in the community system or allocation of caregiver training should be a part of discharge planning to promote recovery.

Keywords: Functional outcome; Modified Rankin; Predictor; Prognostic factor; Risk ratio; Stroke outcome

\section{Introduction}

Stroke is a leading cause of functional impairment, with $20 \%$ of survivors requiring institutional care for 3 months afterwards, and $15 \%$ to $30 \%$ being disabled permanently [1]. Report from the Global Burden of Disease Study 2010 including 188 countries between 1990 and 2013 showed that the absolute number of people affected by stroke in the world over that time period had increased significantly. It was also found that the prevalence nearly doubled for both ischemic and hemorrhagic stroke from 1990 to 2013 [2]. A review in 2016 has shown that over the past 4 decades, a statistical significant trend in stroke incidence rate declined in high-income countries (42\%) but in low- to middle-income countries, it was increasing to greater than $100 \%$ trend over time [3]. In Thailand and other developing countries, changing of lifestyle and dietary results in the increased risk of cardiovascular diseases, particularly stroke. It is estimated that there are 250,000 new stroke cases each year [4]. The consequences after stroke are patients' life-time disability; however, because of a restricted national budget, such developing countries are able to afford a limited budget for the stroke care system, leading to the burden for patients and their families. Therefore, it is important for stroke care teams to be able to identify the variables after an acute care period, which can predict the optimal functional outcome of the patient. A number of studies, including systematic reviews [5-13], have attempted to identify prognostic variables at the functional outcome level in the subacute phase, in order to predict functional recovery from an early stage and consider a proper decision-making process. A systematic review in 2011 by Craig [7] revealed that age, severity of paresis including reduced leg power, presence of hemianopia, size of brain lesion and type of stroke were shown to be predictive factors of mobility outcome. Then in 2015 Meyer et al [8] also performed a systematic review of 27 studies with 63 multivariable mod- 
els and reported predicting functional outcome, as measured by the Barthel Index (BI) or functional independence measure (FIM) after post-stroke rehabilitation. This review found that initial functional level (BI or FIM), National Institute of Health Stroke Scale (NIHSS), dysphagia, impulsivity, neglect (inattention perceptual deficit) and history of previous stroke and age were significant most frequently. However, avalid generalization of the result is still not clearly conclusive, due to many reasons such as the heterogeneity in methodology, especially different outcome measures and timing post stroke. Some studies are often concerned with inpatient rehabilitation $[8,11,12,14-18]$ which might gain benefits from intensive training during hospital admittance. The budget constraints and burden of cost for most individuals during hospitalization cause an early discharge as soon as medical conditions stabilize (i.e. blood pressure, neurological status, etc.). As a result, intensive rehabilitation settings could not be provided widely for all stroke survivors. The aim of this study was to identify predictors related to functional ability in the subacute period within 6 months after discharge from the acute stroke setting. Knowledge of variables that associate with recovery as the final outcome in the short term has been relevant, particularly in continuity of rehabilitation practices after the acute phase and in planning hospital discharge.

\section{Methods}

\section{Subjects}

Retrospective data were reviewed from first-time ischemic stroke patients, who were admitted to the acute stroke at the Faculty of Medicine Hospital, Chiang Mai University from January 2010 to March 2015. Baseline characteristics, demographic data, stroke risk factors (i.e. prior transient ischemic attack (TIA), history of atrial fibrillation (AF), hypertension, dyslipidemia, etc.), co-morbidity and type of stroke were recorded on admission. Other independent variables were considered from previous evidences and clinical backgrounds, including motor assessment, stroke-related consequences, speech problems, facial palsy, dysphagia, perceptual deficit, apraxia, and complications from other causes that might affect recovery were recorded during hospitalization. All stroke-related variables were assessed during the first week after stroke and during 6 months post onset. Motor performance of upper and lower extremities was assessed by manual muscle testing.

\section{Definition of variables and outcome}

Perceptual deficit, unilateral neglect was assessed by observing patients' characteristics (failure to report or respond to stimuli presented to hemiplegic side or failure to perceive their body parts) and confirmed by conventional standardized subtest (i.e. line bisection or copy drawing test) [19]. Apraxia was assessed by observing patients' characteristics, including an inability to perform purposeful movements with one's arms or hands, errors when asked to demonstrate how to use an object or how to carry out actions involving a single or series of components of movements, and problems imitating abstract and symbolic gestures [20]. Speech and communication impairment were assessed via the problems in fluency, comprehension and repetition. Outcomes of this study were modified Rankin Scale (mRS), the measurement of global disability particularly physical disability; and the need for assistance,which was reported as a strong relationship with other clinical measurements of stroke severity and sensitivity in order to identify mild and moderate disability in acute stroke management [10, 21]. All variables and outcomes were assessed and recorded in medical data by certified neurologists or rehabilitation physicians. This study was approved by the Ethics Committee of the Faculty of Medicine, Chiang Mai University, Chiang Mai, Thailand (Research ID: NONE 2558-03123) on July 24, 2015.

\section{Statistical analysis}

Descriptive statistics (frequency, percentage, mean and standard deviation) were used to describe the baseline characteristics and stroke risk factors. The Fisher's exact test or Student's $t$ test was performed where appropriate for comparison between groups, with a significance level of $\mathrm{P}<0.05$. To develop a prediction model, the step-backward method of multivariable risk regression analysis was used with the generalized linear model to derive independent predictive variables. Firstly, all statistically significant demographic variables, pre-stroke risk factors and post-stroke complications with $\mathrm{P}<0.05$ from univariable analysis or meaningful clinical variables were submitted to the multivariable analysis model. The final independent predictive variables from second backward stepwise regression were shown with the risk ratio (RR) and 95\% confident interval $(\mathrm{CI})$ in which $\mathrm{P}<0.05$ indicated statistical significance. Functional recovery group was defined as the mRS score of 13 (non-significant disability to moderate disability, but able to walk unassisted), whereas a score of 4 - 5 (moderately severe disability to severe disability) was defined as non-functional recovery group. All statistical analyses were performed with Stata software.

\section{Results}

Of 358 first-time ischemic stroke patients, who were admitted at the stroke unit from January 2010 to March 2015 and recruited into this study, $255(71.2 \%)$ were classified as the functional recovery group (mRS score of 1 - 3) within 6 months after onset and $103(28.8 \%)$ were non-functional recovery group (mRS score of 4 - 5), respectively [21]. Baseline demographics and clinical data among the two groups are shown in Table 1. Some variables were found to have statistical difference between the groups; the non-functional recovery group was more likely to have large artery atherosclerosis subtype, lower initial Glasgow coma scale (GCS) on hospital admission, higher prevalence of prior TIA, AF and other co-morbidities, and be at an old age.

When considering motor performance within 6 months 
Table 1. Demographic Data, Premorbid Health Status and Stroke-Related Characteristics According to Functional Recovery at Baseline $(n=358)$

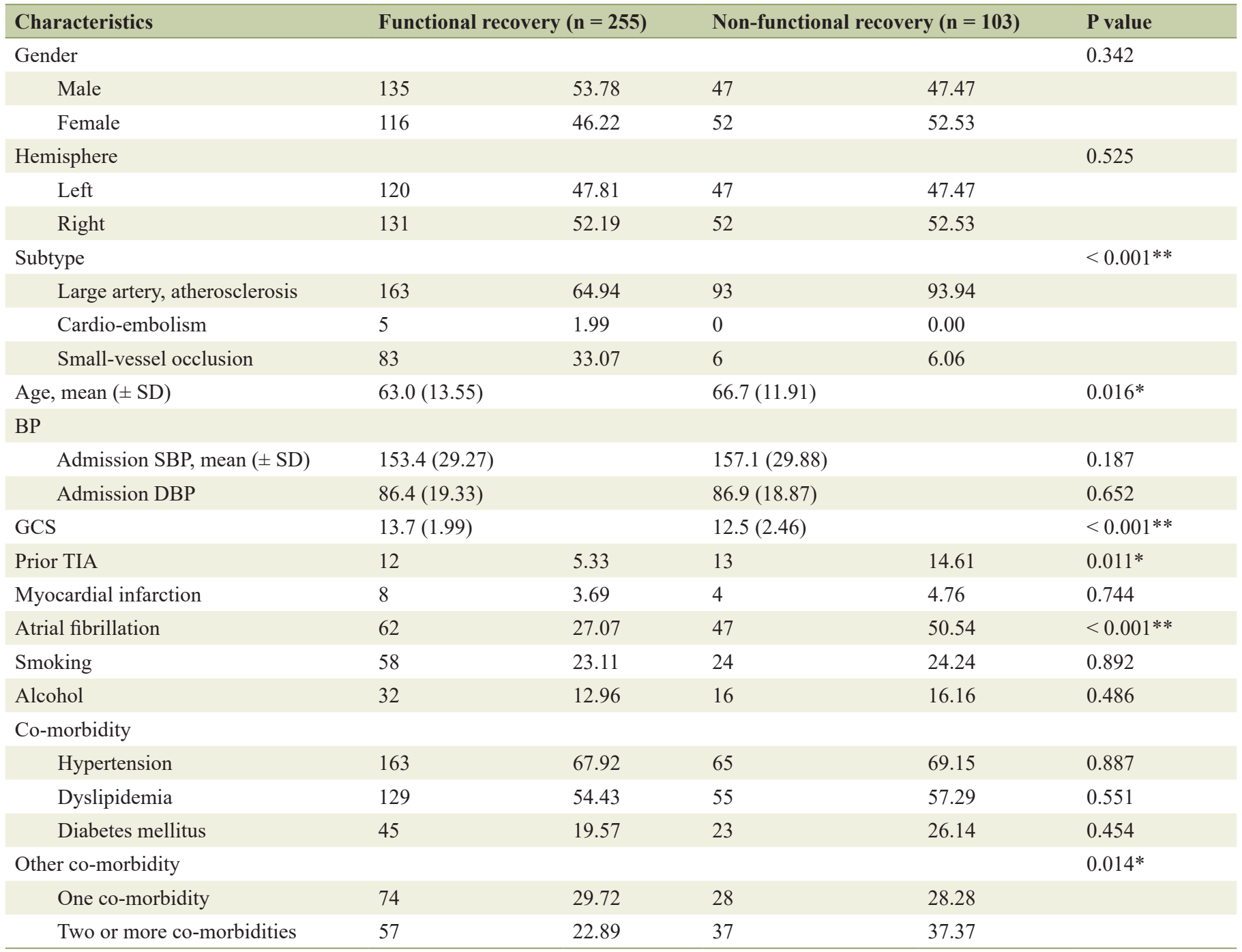

GCS: Glasgow coma scale; TIA: transient ischemic attack. ${ }^{*} \mathrm{P}<0.05,{ }^{* *} \mathrm{P}<0.001$.

post onset and stroke-related complications, it was found that motor performance of the hemiparetic arm and leg, communication and swallowing dysfunction and other complications, besides those that are stroke-related, were different statistically between patients in the functional recovery group and those in the non-functional one (Table 2).

\section{Prognostic factors for post-acute stage after stroke}

By univariable analysis, age of over 75 years $(\mathrm{P}=0.043)$, prior TIA $(\mathrm{P}=0.026)$, history of AF $(\mathrm{P}<0.001)$ and other comorbidities $(\mathrm{P}=0.016)$, initial GCS on hospital admission ( $\mathrm{P}$ $=0.001)$, motor arm and leg score $(\mathrm{P}<0.001)$, aphasia $(\mathrm{P}=$ $0.008)$, dysphagia $(\mathrm{P}=0.034)$ and other complications besides those that are stroke-related $(\mathrm{P}<0.001)$ were found to associate with functional gain and entrance to the multivariable analysis generalized linear model (Table 3).

Significant variables from univariable analysis were then submitted to the multivariable analysis. Results of the multivariable analyses are shown in Table 4 . The independent predictors for functional recovery in this setting were leg score of motor assessment within 6 months after onset with a risk ratio $(\mathrm{RR}=1.92,95 \% \mathrm{CI}: 1.14-3.21, \mathrm{P}=0.013)$ followed by arm score $(\mathrm{RR}=1.75,95 \% \mathrm{CI}: 1.02-3.01, \mathrm{P}=0.042)$ and age $(\mathrm{RR}$ $=1.36,95 \% \mathrm{CI}: 1.04-1.77, \mathrm{P}=0.025)$.

\section{Discussion}

Most developing countries experienced the economic constraint thus limiting healthcare budget and leading to a trend of shortened hospital stay as well as an increasing demand for efficiency in the continuity of stroke care. This implies that 
Table 2. Comparison of Motor Assessment and Post-Stroke Complications Between the Independent Functional Recovery Group $(n=255)$ and Non-Functional Recovery Group $(n=103)$

\begin{tabular}{|c|c|c|c|c|c|}
\hline \multirow{2}{*}{ Characteristics } & \multicolumn{2}{|c|}{ Functional recovery } & \multicolumn{2}{|c|}{ Non-functional recovery } & \multirow{2}{*}{ P value } \\
\hline & n & $\%$ & n & $\%$ & \\
\hline \multicolumn{6}{|l|}{ Follow-up motor assessment } \\
\hline Grade 0 - 1 & 95 & 24.70 & 70 & 70.71 & \\
\hline Grade 2 - 3 & 94 & 37.45 & 24 & 24.24 & \\
\hline Grade 0 - 1 & 47 & 18.73 & 62 & 62.63 & \\
\hline Grade 2 - 3 & 102 & 40.64 & 31 & 31.31 & \\
\hline More than $3+$ & 102 & 40.64 & 6 & 6.06 & \\
\hline \multicolumn{6}{|l|}{ Stroke-related complications } \\
\hline Global aphasia & 24 & 9.56 & 24 & 9.28 & \\
\hline Dysphagia & 10 & 4.00 & 11 & 24.74 & $0.033^{*}$ \\
\hline Inattention & 14 & 5.60 & 11 & 11.22 & 0.107 \\
\hline Apraxia & 4 & 1.57 & 1 & 0.97 & 1.000 \\
\hline Depression & 9 & 3.60 & 9 & 9.18 & $0.042 *$ \\
\hline Other complications & & & & & $<0.001^{* *}$ \\
\hline One complication & 50 & 20.08 & 24 & 24.24 & \\
\hline Two or more complications & 21 & 8.43 & 23 & 23.23 & \\
\hline
\end{tabular}

MMT: manual muscle testing; ${ }^{*} \mathrm{P}<0.05,{ }^{*} \mathrm{P}<0.001$.

knowledge about the prognosis for outcome regarding basic functional activities and mobility is of particular importance in optimizing stroke management [22]. The findings from this study seem to be consistent with previous prognostic studies, in

Table 3. Independent Predictors of Functional Recovery Within 6 Months Post Stroke From the Univariable Analysis Generalized Linear Model

\begin{tabular}{llll}
\hline Predictors & RR & $\mathbf{9 5 \%}$ CI & P value \\
\hline Age over 75 years & 1.30 & $1.00-1.69$ & $0.043^{*}$ \\
Prior TIA & 1.98 & $1.10-3.56$ & $0.026^{*}$ \\
Initial GCS $<12$ & 2.02 & $1.35-3.03$ & $0.001^{*}$ \\
Atrial fibrillation & 1.99 & $1.32-2.99$ & $<0.001^{* *}$ \\
Co-morbidity & 1.32 & $1.05-1.68$ & $0.016^{*}$ \\
Motor arm & 2.95 & $2.11-4.11$ & $<0.001^{* *}$ \\
Motor leg & 2.84 & $2.09-3.87$ & $<0.001^{* *}$ \\
Aphasia & 1.38 & $1.09-1.76$ & $0.008^{*}$ \\
Dysphagia & 1.97 & $1.05-3.69$ & $0.034^{*}$ \\
Other complications & 1.51 & $1.19-1.92$ & $<0.001^{* *}$ \\
\hline
\end{tabular}

RR: risk ratio; $\mathrm{Cl}$ : confidence interval. ${ }^{*} \mathrm{P}<0.05,{ }^{* *} \mathrm{P}<0.001$. that arm and leg motor ability [6, 8, 12, 16, 18, 23, 24] and age $[8,10,17,18,25-28]$ have been identified as potential predictors of functional outcome. Based on a review by Hakkennes [18], most frequent predictors relating to acute hospital discharge were age, presence of hemiparesis, severity of impairment, and cognition and functional level post stroke. This study also found that motor improvement of the leg and arm and older age were independent predictors of functional outcome during 6 months post onset $(\mathrm{RR}$ of leg $=1.92, \mathrm{RR}$ of $\mathrm{arm}=1.75$, and $\mathrm{RR}$ of age $=1.36 ; \mathrm{P}<0.05)$. Regained use of the upper extremities was a predictor for hand function and activities of daily living (ADL) domains, and that of lower extremity motor function was a positive predictor for the functional mobility domain and

Table 4. Independent Predictors of Functional Recovery Within 6 Months Post Stroke Using the Multivariable Analysis Generalized Linear Model

\begin{tabular}{llll}
\hline Predictors & RR & $\mathbf{9 5 \%}$ CI & P value \\
\hline Motor leg & 1.92 & $1.14-3.21$ & $0.013^{*}$ \\
Motor arm & 1.75 & $1.02-3.01$ & $0.042^{*}$ \\
Age more than 75 & 1.36 & $1.04-1.77$ & $0.025^{*}$ \\
\hline
\end{tabular}

RR: risk ratio; Cl: confidence interval. ${ }^{*} \mathrm{P}<0.05$. 
total scores of health-related quality of life (HRQOL) outcomes [16]. Neural plasticity, the process of brain reorganization to compensate for injury, relies on a time-dependent mechanism, as it is increased over early weeks and decreased over months after a stroke, and the last step of recovery is based mainly on rehabilitative training $[29,30]$. The findings of this study supported the importance of rehabilitative training strategies toward improving motor performance in the subacute period to enhance the mechanism of neuroplasticity [29, 30]. Although report recently from the Cochrane Database Systematic Review [31] could not conclude and support the positive effect of caregiver training on functional outcome after stroke, in our perspective for such limited rehabilitation resources in some countries, promoting continuity of rehabilitation in the community system and caregiver training should still be a part of discharge planning to help continuing the program for stroke.

It was not surprising that elderly people with a stroke are a negative factor when associated with functional gain. Age was one of the most frequent factors found to associate with poor functional outcome in a population-based study [11] and review [8]. It was suggested that when developing a multivariable model in functional outcome, age should be included in all of the identified models, and one of the variables that should be considered most in clinical practice [8]. Older patients were more likely to be discharged to accommodation with support rather than be discharged home [18]. Due to physiological changes, older individuals may have more cognitive deficits, more co-morbidity, and may experience strokes that impact different regions from those of younger patients [8], and care should be taken in elderly stroke patients, as they are at high risk of falling and other complications.

Some variables were not addressed in the final model of multivariable analysis: the presence of AF, prior TIA, comorbidities, complications during admission, initial GCS less than 12 and consequences of stroke complications; aphasia and dysphagia were found to have association with functional recovery in univariable analysis. These factors were found to be significantly different among the functional and non-functional recovery groups (Tables 1,2 ). The presence of AF is a known factor affecting the ability of patients to exercise, and it was found that patients with $\mathrm{AF}$ were more likely to develop cardiac complications and it is also the cause of death from non-neurologic reasons [32]. However, the role of AF in functional outcome remains controversial, as evidenced by Mizrahi et al [33], who demonstrated from multiple linear regression, that despite discharge, the FIM score in non-AF was significantly higher when compared with AF patients, and AF was not found to be an independent predictor of functional gain during the rehabilitation period [33]. Prior TIA, or having a number of co-morbidities in addition to stroke risk factors, was found to relate to not achieving functional goals in some studies $[18,28,34]$, which is consistent with the results of this study. The number of co-morbidities reflects health condition prior to stroke, including chronic medical issues such as chronic kidney disease, gout and degenerative arthritis, which may involve physical deficit due to stroke.

Aphasia and dysphagia were seen to associate with poor functional outcome in the univariable analysis. Global aphasia was the most serious communication problem found in $1 / 4$
$(24.74 \%)$ of the non-functional recovery group. This more likely supported earlier evidence that a severe type of aphasia is associated with the lower rate of functional recovery and HRQOL in stroke survivors [11, 26, 35-37]. Dysphagia, or difficulty in swallowing, was found to be one of the most frequent significant predictors of functional outcome by either the BI or FIM in a 2015 review $[8,38]$, as well as being reported for having increased association with pulmonary complications. In a review by Martino et al [39], stroke patients with dysphagia had increased risk of pneumonia $(\mathrm{RR}=3.17,95 \% \mathrm{CI}: 2.07$ - 4.87) and aspiration ( $R R=11.56,95 \%$ CI: 3.36 - 39.77). It was reported as the even greater risk for elderly stroke patients, as aging causes changes in muscle strength of the masticatory apparatus [39-41].

Although explored infrequently, univariable analysis identified patients with an initial GCS of less than 12, and those with other complications regarding stroke-related complications were a negative predictor for achieving functional improvement. Coma, impaired consciousness at hospital admission, was an important predictor of complications and short-term mortality [32, 42]. More medical complications post stroke, besides neurological complications, are associated with poor recovery in several studies [18, 28, 42, 43]. The most frequent complication found in this study was aspiration pneumonia, which is consistent with previous study [42].

Limitations

The limitations of this study result from its retrospective nature. The data might rely on the expertise of individual physicians, and perhaps some variables were omitted in some records causing an incomplete statistical analysis in some aspects. Based on clinical implication, cognitive impairment following stroke is common among older stroke patients, and the more severe the impairment is, the greater the effect on functional outcome would be, and measures of both physical and cognitive function were recommended in studies of stroke outcome $[44,45]$. Balance at admission, as measured with the Berg Balance Scale, was found to have the strongest association with discharge functioning [45], and is a basic component needed for functional mobility, namely doing activities while sitting, transferring to a wheelchair and walking. Indeed, the initial ability to control the trunk while sitting at acute phase was an early predictor for subacute functional outcome [25, 46, 47]. Psychological or socio-demographic factors such as motivation, or patient and family background might have provided extra impact on functional status [48]. Finally, most of the patients in this study were from the acute stroke unit and were discharged after their medical status had stabilized. Therefore, shorter length of hospital stay as well as not receiving rehabilitative treatment from a professional rehabilitation team may result in worse outcome [28,49]. Due to this limitation, interpretation should be taken with caution.

\section{Conclusions}

The independent predictors found in this study for functional recovery during 6 months post stroke were age and motor improvement, which is consistent with previous literature. Although our study did not shed a new light on prediction for 
functional recovery in stroke, it depicted and confirmed the previous results from other studies but in Thai population.

\section{Acknowledgments}

The authors would like to thank Professor Jayanthorn Patumanond and Assistant Professor Chidchanok Ruengorn for their contribution in data analysis.

\section{Funding}

This study has no funding support.

\section{Conflict of Interest}

The authors declare that they have no conflict of interest.

\section{Informed Consent}

Not applicable.

\section{Author Contributions}

SK developed the theoretical and conceptual framework, collected data, performed statistical analysis of the results and wrote manuscript with supported from JK. JK supervised the whole research, and guided in preparing scope of manuscript and final proofreading and approval of the version for publication. PW contributed to planning data collecting process, data interpretation and writing the final manuscript. KW conceived the idea and advised SK in developing case record form, and contributed to planning data collecting process. JK, PW and $\mathrm{KW}$ aided in interpreting the results and provided feedback and suggestion for the final manuscript.

\section{Data Availability}

The dataset used to support the findings of this study is restricted by the Ethics Committee of the Faculty of Medicine, Chiang Mai University, Chiang Mai, Thailand in order to protect patient privacy. Data are available from Siriphan Kongsawasdi, Clinical Epidemiology Program, Faculty of medicine, Chiang Mai University for researchers who meet the criteria for access to confidential data.

\section{Disclosure}

This work was based on a $\mathrm{PhD}$ thesis (Siriphan Kongsawasdi) from the Chiang Mai University, Thailand. This study was presented as the poster session at the World Congress of Neurol- ogy (WCN 2017) https://www.jns-journal.com/article/S0022$510 X(17) 33724-3 /$ pdf.

\section{References}

1. Writing Group M, Lloyd-Jones D, Adams RJ, Brown TM, Carnethon M, Dai S, De Simone G, et al. Heart disease and stroke statistics - 2010 update: a report from the American Heart Association. Circulation. 2010;121(7):e46-e215.

2. Feigin VL, Krishnamurthi RV, Parmar P, Norrving B, Mensah GA, Bennett DA, Barker-Collo S, et al. Update on the global burden of ischemic and hemorrhagic stroke in 1990-2013: the GBD 2013 study. Neuroepidemiology. 2015;45(3):161-176.

3. Thrift AG, Thayabaranathan T, Howard G, Howard VJ, Rothwell PM, Feigin VL, Norrving B, et al. Global stroke statistics. Int J Stroke. 2017;12(1):13-32.

4. Suwanwela NC. Stroke epidemiology in Thailand. J Stroke. 2014;16(1):1-7.

5. Abdul-sattar AB, Godab T. Predictors of functional outcome in Saudi Arabian patients with stroke after inpatient rehabilitation. NeuroRehabilitation. 2013;33(2):209-216.

6. Brown AW, Therneau TM, Schultz BA, Niewczyk PM, Granger CV. Measure of functional independence dominates discharge outcome prediction after inpatient rehabilitation for stroke. Stroke. 2015;46(4):1038-1044.

7. Craig LE, Wu O, Bernhardt J, Langhorne P. Predictors of poststroke mobility: systematic review. Int J Stroke. 2011;6(4):321-327.

8. Meyer MJ, Pereira S, McClure A, Teasell R, Thind A, Koval J, Richardson M, et al. A systematic review of studies reporting multivariable models to predict functional outcomes after post-stroke inpatient rehabilitation. Disabil Rehabil. 2015;37(15):1316-1323.

9. Ostwald SK, Swank PR, Khan MM. Predictors of functional independence and stress level of stroke survivors at discharge from inpatient rehabilitation. J Cardiovasc Nurs. 2008;23(4):371-377.

10. Reid JM, Dai D, Christian C, Reidy Y, Counsell C, Gubitz GJ, Phillips SJ. Developing predictive models of excellent and devastating outcome after stroke. Age Ageing. 2012;41(4):560-564.

11. Scrutinio D, Monitillo V, Guida P, Nardulli R, Multari V, Monitillo F, Calabrese G, et al. Functional gain after inpatient stroke rehabilitation: correlates and impact on long-term survival. Stroke. 2015;46(10):2976-2980.

12. Ten Brink AF, Hajos TR, van Bennekom C, Nachtegaal J, Meulenbelt HE, Fleuren JF, Kouwenhoven M, et al. Predictors of physical independence at discharge after stroke rehabilitation in a Dutch population. Int J Rehabil Res. 2017;40(1):37-45.

13. Veerbeek JM, Kwakkel G, van Wegen EE, Ket JC, Heymans MW. Early prediction of outcome of activities of daily living after stroke: a systematic review. Stroke. 2011;42(5):1482-1488.

14. Masiero S, Avesani R, Armani M, Verena P, Ermani M. Predictive factors for ambulation in stroke patients in the rehabilitation setting: a multivariate analysis. Clin Neurol 
Neurosurg. 2007;109(9):763-769.

15. Singh R, Hunter J, Philip A, Todd I. Predicting those who will walk after rehabilitation in a specialist stroke unit. Clin Rehabil. 2006;20(2):149-152.

16. Chen CM, Tsai CC, Chung CY, Chen CL, Wu KP, Chen HC. Potential predictors for health-related quality of life in stroke patients undergoing inpatient rehabilitation. Health Qual Life Outcomes. 2015;13:118.

17. Luk JK, Cheung RT, Ho SL, Li L. Does age predict outcome in stroke rehabilitation? A study of 878 Chinese subjects. Cerebrovasc Dis. 2006;21(4):229-234.

18. Hakkennes SJ, Brock K, Hill KD. Selection for inpatient rehabilitation after acute stroke: a systematic review of the literature. Arch Phys Med Rehabil. 2011;92(12):20572070.

19. Yang NY, Zhou D, Chung RC, Li-Tsang CW, Fong KN. Rehabilitation interventions for unilateral neglect after stroke: a systematic review from 1997 through 2012. Front Hum Neurosci. 2013;7:187.

20. Perez-Marmol JM, Garcia-Rios MC, Barrero-Hernandez FJ, Molina-Torres G, Brown T, Aguilar-Ferrandiz ME. Functional rehabilitation of upper limb apraxia in poststroke patients: study protocol for a randomized controlled trial. Trials. 2015;16:508.

21. Banks JL, Marotta CA. Outcomes validity and reliability of the modified Rankin scale: implications for stroke clinical trials: a literature review and synthesis. Stroke. 2007;38(3):1091-1096.

22. Kwakkel G, Kollen BJ. Predicting activities after stroke: what is clinically relevant? Int J Stroke. 2013;8(1):25-32.

23. Shelton FD, Volpe BT, Reding M. Motor impairment as a predictor of functional recovery and guide to rehabilitation treatment after stroke. Neurorehabil Neural Repair. 2001;15(3):229-237.

24. Weimar C, Ziegler A, Konig IR, Diener HC. Predicting functional outcome and survival after acute ischemic stroke. J Neurol. 2002;249(7):888-895.

25. Carod-Artal FJ, Medeiros MS, Horan TA, Braga LW. Predictive factors of functional gain in long-term stroke survivors admitted to a rehabilitation programme. Brain Inj. 2005;19(9):667-673.

26. Gialanella B, Bertolinelli M, Lissi M, Prometti P. Predicting outcome after stroke: the role of aphasia. Disabil Rehabil. 2011;33(2):122-129.

27. Lin JH, Hsieh CL, Lo SK, Hsiao SF, Huang MH. Prediction of functional outcomes in stroke inpatients receiving rehabilitation. J Formos Med Assoc. 2003;102(10):695700 .

28. O'Brien SR, Xue Y. Predicting goal achievement during stroke rehabilitation for Medicare beneficiaries. Disabil Rehabil. 2014;36(15):1273-1278.

29. Dimyan MA, Cohen LG. Neuroplasticity in the context of motor rehabilitation after stroke. Nat Rev Neurol. 2011;7(2):76-85.

30. Hara Y. Brain plasticity and rehabilitation in stroke patients. J Nippon Med Sch. 2015;82(1):4-13.

31. Vloothuis JD, Mulder M, Veerbeek JM, Konijnenbelt M, Visser-Meily JM, Ket JC, Kwakkel G, et al. Caregivermediated exercises for improving outcomes after stroke.
Cochrane Database Syst Rev. 2016;12:CD011058.

32. Alonso A, Ebert AD, Kern R, Rapp S, Hennerici MG, Fatar M. Outcome Predictors of Acute Stroke Patients in Need of Intensive Care Treatment. Cerebrovasc Dis. 2015;40(1-2):10-17.

33. Mizrahi EH, Fleissig Y, Arad M, Adunsky A. Short-term functional outcome of ischemic stroke in the elderly: a comparative study of atrial fibrillation and non-atrial fibrillation patients. Arch Gerontol Geriatr. 2014;58(1):121124.

34. Yeh HJ, Huang N, Chou YJ, Cheng SP, Lee WK, Lai CC, Cheng CC. Older Age, Low Socioeconomic Status, and Multiple Comorbidities Lower the Probability of Receiving Inpatient Rehabilitation Half a Year After Stroke. Arch Phys Med Rehabil. 2017;98(4):707-715.

35. Gialanella B, Ferlucci C. Functional outcome after stroke in patients with aphasia and neglect: assessment by the motor and cognitive functional independence measure instrument. Cerebrovasc Dis. 2010;30(5):440-447.

36. Tur BS, Gursel YK, Yavuzer G, Kucukdeveci A, Arasil T. Rehabilitation outcome of Turkish stroke patients: in a team approach setting. Int J Rehabil Res. 2003;26(4):271277.

37. Boehme AK, Martin-Schild S, Marshall RS, Lazar RM. Effect of aphasia on acute stroke outcomes. Neurology. 2016;87(22):2348-2354.

38. Meijer R, Ihnenfeldt DS, de Groot IJ, van Limbeek J, Vermeulen M, de Haan RJ. Prognostic factors for ambulation and activities of daily living in the subacute phase after stroke. A systematic review of the literature. Clin Rehabil. 2003;17(2):119-129.

39. Martino R, Foley N, Bhogal S, Diamant N, Speechley M, Teasell R. Dysphagia after stroke: incidence, diagnosis, and pulmonary complications. Stroke. 2005;36(12):27562763.

40. Lugger KE. Dysphagia in the elderly stroke patient. J Neurosci Nurs. 1994;26(2):78-84.

41. Oliveira AR, Costa AG, Morais HC, Cavalcante TF, Lopes MV, Araujo TL. Clinical factors predicting risk for aspiration and respiratory aspiration among patients with Stroke. Rev Lat Am Enfermagem. 2015;23(2):216-224.

42. Mamushet Y, Zenebe G, Addissie A. Medical and Neurological Complications among Stroke Patients Admitted for Inpatient Care in Addis Ababa, Ethiopia. Ethiop Med J. 2015;53(1):9-17.

43. Janus-Laszuk B, Mirowska-Guzel D, Sarzynska-Dlugosz I, Czlonkowska A. Effect of medical complications on the after-stroke rehabilitation outcome. NeuroRehabilitation. 2017;40(2):223-232.

44. Patel MD, Coshall C, Rudd AG, Wolfe CD. Cognitive impairment after stroke: clinical determinants and its associations with long-term stroke outcomes. J Am Geriatr Soc. 2002;50(4):700-706.

45. Wee JY, Hopman WM. Stroke impairment predictors of discharge function, length of stay, and discharge destination in stroke rehabilitation. Am J Phys Med Rehabil. 2005;84(8):604-612.

46. Oh HM, Im S, Ko YA, Ko SB, Park GY. The sitting-unsupported balance score as an early predictor of function- 
al prognosis in stroke patients: a pilot study. Ann Rehabil Med. 2013;37(2):241-246.

47. Kim TJ, Seo KM, Kim DK, Kang SH. The relationship between initial trunk performances and functional prognosis in patients with stroke. Ann Rehabil Med. 2015;39(1):6673.

48. Stucki G, Cieza A, Melvin J. The International Classifica- tion of Functioning, Disability and Health (ICF): a unifying model for the conceptual description of the rehabilitation strategy. J Rehabil Med. 2007;39(4):279-285.

49. Duncan PW, Horner RD, Reker DM, Samsa GP, Hoenig H, Hamilton B, LaClair BJ, et al. Adherence to postacute rehabilitation guidelines is associated with functional recovery in stroke. Stroke. 2002;33(1):167-177. 